COMMUNICATIONS IN

ANALYSIS AND GEOMETRY

Volume 10, Number 1, 127-150, 2002

\title{
Calibrated Fibrations
}

\author{
EDWARD GOLDSTEIN
}

\begin{abstract}
In this paper we investigate various properties of calibrated submanifolds and of Special Lagrangian fibrations. Our main result is a construction of a Special Lagrangian fibration on a certain compact, simply-connected almost Calabi-Yau threefold- we believe this to be the first known example of such a fibration.
\end{abstract}

\section{Introduction.}

The notion of a calibration was introduced by Harvey and Lawson in their seminal paper [3]. Let $M$ be a Riemannian manifold and let $\eta$ be a closed $k$-form on $M$. We say that $\eta$ is a calibration on $M$ if for any oriented $k$-plane $\kappa$ in the tangent bundle of $M,\left.\eta\right|_{\kappa} \leq \operatorname{vol}(\kappa)$ (here $\operatorname{vol}(\kappa)$ is the volume form on $\kappa$ ). An oriented $k$-dimensional submanifold $L$ of $M$ is said to be calibrated by $\eta$ if $\eta$ restricts to the volume form on $L$. Calibrated submanifolds are homologically volume minimizing (see [3], Theorem II.4.2). Harvey and Lawson have also investigated the fundamental calibrated geometries in the Euclidean space.

R.C. McLean had studied the deformation theory of calibrated submanifolds in [6]. He showed that in several cases the deformation theory is unobstructed and the moduli-space of calibrated submanifolds is a smooth manifold. This raises a natural question about a connection between the global properties of the moduli-space of calibrated submanifolds and the geometry of the ambient manifold. One instance of such a question is the SYZ conjecture (see [11]), which suggests in particular that a Calabi-Yau manifold has a fibration with generic fiber being a Special Lagrangian torus. In this paper we take some steps in investigating the geometry of calibrated submanifolds and the global structure of their moduli-space. The paper is organized as follows:

In section 2 we prove a comparison theorem for volumes of small balls in a calibrated submanifold of a Riemannian manifold $M$. Suppose the sectional curvatures of $M$ are bounded from above by some constant $K$. Let $L$ be a minimal submanifold in $M, p \in L$ a point and $B(p, r)$ be a ball of radius $r$ around $p$ in $M$. There are a number of results on comparison between the 
volume of $L \bigcap B(p, r)$ and the volume of a ball of radius $r$ in a space form of constant curvature $K$. Our main result in section 2 is Theorem 2.0.2, which states that if $L$ is a calibrated $k$-dimensional submanifold of $M$ then the volume of a ball of radius $r$ in the induced metric on $L$ (which is smaller than $L \bigcap B(p, r))$ is greater than the volume of a ball of the same radius in a $k$-dimensional space form of constant curvature $K$ for $r \leq r_{0}$ with $r_{0}$ depending only on the ambient manifold $M$. As a corollary we deduce that if $\eta$ is a calibrating $k$-form on $M$ and $\beta \in H_{k}(M, \mathbb{Z})$ is a homology class then there is an a-priori bound on a diameter of submanifolds of $M$ calibrated by $\eta$ representing the homology class $\beta$.

In section 3 we study one example of a calibrated geometry : the Special Lagrangian geometry on almost Calabi-Yau manifolds. Let $\left(M^{2 n}, \omega\right)$ be a Kahler manifold with a non-vanishing holomorphic $(n, 0)$-form $\varphi$ on $M$. We will call $(M, \omega, \varphi)$ an almost Calabi-Yau manifold. $(M, \omega, \varphi)$ is a Calabi-Yau manifold if $\varphi$ has constant length $(\sqrt{2})^{n}$. In this case one easily shows that $\varphi$ is parallel, the metric is Ricci-flat and the holonomy of $M$ is contained in $S U(n)$.

If $\left(M^{2 n}, \omega, \varphi\right)$ is a compact almost Calabi-Yau manifold then Yau's resolution of the Calabi conjecture (see [13]) gives a unique Kahler metric $\omega_{C Y}$ in the same cohomology class as $\omega$ s.t. $\varphi$ has constant length $C$ with respect to $\omega_{C Y}$. We can multiply $\varphi$ by a constant to get $C=(\sqrt{2})^{n}$. Thus $\left(M, \omega_{C Y}, \varphi\right)$ becomes a Calabi-Yau manifold and $\omega_{C Y}$ is called a Calabi-Yau metric.

Let $V$ be a complex vector space of complex dimension $n$ with a Hermitian 2-form $\omega_{0}$. Let $\varphi_{0}$ be an element of $\Lambda^{(n, 0)} V^{*}$ s.t. its length $\left|\varphi_{0}\right|=(\sqrt{2})^{n}$ with respect to the Hermitian metric defined by $\omega_{0}$. Harvey and Lawson have observed (see [3], Corollary III.1.11) that the $n$-form $\operatorname{Re} \varphi_{0}$, viewed as a constant $n$-form on the linear manifold $V$ is a calibration on $V$. Moreover a submanifold $L \subset V$ is calibrated iff $\left.\omega_{0}\right|_{L}=0,\left.\operatorname{Im} \varphi_{0}\right|_{L}=0$. Those submanifolds are called Special Lagrangian (SLag) submanifolds.

The notion of SLag submanifolds readily generalizes to almost CalabiYau manifolds. Let $(M, \omega, \varphi)$ be an almost Calabi-Yau manifold. We can conformally scale the Kahler form $\omega$ to a Hermitian 2-form $\omega^{*}$ on $M$ so that the form $\varphi$ will have length $(\sqrt{2})^{n}$ with respect to the metric defined by $\omega^{*}$. Since $\omega^{*}$ defines a Hermitian metric $g^{*}$ on $M$ and $\varphi$ has constant length $(\sqrt{2})^{n}$ with respect to this metric, the form $\operatorname{Re} \varphi$ will be a calibrating $n$-form on $\left(M, g^{*}\right)$. Following Harvey and Lawson we define SLag submanifolds to be submanifolds calibrated by $\operatorname{Re} \varphi$ on $\left(M, g^{*}\right)$ (see [3], p. 153). Thus a submanifold $L \subset M$ is a SLag submanifold iff $\left.\omega^{*}\right|_{L}=0$ and $\left.\operatorname{Im} \varphi\right|_{L}=0$, 
which is of course equivalent to

$$
\left.\omega\right|_{L}=0,\left.\operatorname{Im} \varphi\right|_{L}=0
$$

Let $L$ be a (compact) SLag submanifold of an almost Calabi-Yau manifold $(M, \omega, \varphi)$. Lemma 3.1.1 shows (generalizing McLean's Corollary 3.9 of [6] for a Calabi-Yau metric) that the moduli-space $\Phi$ of SLag submanifolds of $M$ passing through $L$ is smooth of dimension equal to the first Betti number of $L$. We will see that one can use automorphisms of $(M, \omega, \varphi)$ to understand some global properties of $\Phi$ (see Lemmas 3.1.2-3.1.3).

If $b_{1}(L)=n$ one can ask if the moduli-space of SLag submanifolds through $L$ gives a fibration of some neighbourhood of $L$ in $M$. Now SLag submanifolds are in particular Lagrangian submanifolds of $(M, \omega)$. If one has a fibration of some open subset of a symplectic manifold by compact Lagrangian submanifolds, then Duistermaat's theory of action-angle coordinates suggests in particular that those submanifolds are diffeomorphic to a torus (see [2] and [1], section 2). So tori are the only candidates for being fibers of a SLag fibration on an open subset of $M$.

Suppose we have a SLag torus fibration on some open subset of $M$. We would like to see when this fibration extends to a fibration over the whole of $M$. Now there are obvious topological obstructions for that. Also there are geometric obstructions for the compactness of a moduli-space of SLag submanifolds (see Lemma 3.1.3). Thus we can't in general expect an "honest" SLag torus fibration on $M$. We make the following definition:

Definition 1.0.1. Let $\left(M^{2 n}, \omega, \varphi\right)$ be an almost Calabi-Yau manifold. A SLag fibration for $M$ is a surjective map $\alpha$ onto a topological space $S$ and an open subset $S_{0} \subset S$ s.t.

1) $S_{0}$ is a smooth $n$-dimensional manifold and the map $\alpha: M_{0}=$ $\alpha^{-1}\left(S_{0}\right) \mapsto S_{0}$ is a smooth fibration with fibers being SLag tori in $M$

2) The complement $M-M_{0}$ (the total space of singular fibers) is contained in the image $i: N \mapsto M$ of a smooth map $i$ from a compact, $(2 n-2)$ dimensional manifold $N$ into $M$.

If $(M, \omega, \varphi)$ is a Calabi-Yau manifold then the Strominger-Yau-Zaslow (SYZ) conjecture (see [11]) suggests that $M$ has a SLag fibration (see Definition 1.0.1). This conjecture naturally generalizes to almost Calabi-Yau manifolds. The conjecture also proposes an approach to constructing a mirror Calabi-Yau manifold to $M$ using this SLag fibration (see [1] for some progress on constructing a mirror assuming that the SLag fibration on $M$ 
exists). We will see in Lemma 3.1.4 that the singular fibers of a SLag torus fibration are related to the fixed points of automorphisms of $(M, \omega, \varphi)$.

In section 3.2 we study one example of an almost Calabi-Yau threefold in detail: the Borcea-Voisin threefold $M$. We define a certain Kahler metric $\omega$ on $M$ and we study a moduli-space $\Phi$ of SLag tori with respect to this metric. We will characterize the singular SLag subvarieties, to which the elements of $\Phi$ degenerate (those will be a product of a circle with a cusp curve). We consider the compactified moduli-space $\bar{\Phi}$ of SLag subvarieties in $M$. We will see that different elements of $\bar{\Phi}$ don't intersect in $M$. Moreover we prove that the boundary $\bar{\Phi}-\Phi$ will be parameterized by a finite union of circles. This will enable us to prove that $\bar{\Phi}$ fills $M$. More precisely we get a SLag fibration $\alpha$ from $M$ onto $\bar{\Phi}$ according to Definition 1.0.1 with the generic fiber (in $\Phi$ ) being a SLag torus in $M$. As far as we know this is a first example of a compact, simply connected almost Calabi-Yau threefold, which admits a SLag fibration (though the fibration is SLag with respect to a Kahler metric, which is not Ricci-flat).

In section 3.3 we will examine holomorphic functions on an almost Calabi-Yau manifold in a neighbourhood of a SLag submanifold. An immediate consequence of the fact that SLag submanifolds are 'Special' is Theorem 3.3.1, which states that the integral of a holomorphic function over SLag submanifolds is a constant function on their moduli-space. This gives a restriction on how a family of SLag submanifolds might approach a singularity (Corollary 3.3.1) and also shows that smooth SLag submanifolds asymptotic to a cone in $\mathbb{C}^{n}$ can't be too "thin" (Lemma 3.3.1).

Acknowledgments. This paper constitutes a part of author's Ph.D. at the Massachusetts Institute of Technology. The author wants to thank his advisor, Tom Mrowka, for initiating him into the subject and for continuing support. He is also grateful to Gang Tian for a number of useful conversations.

\section{Volume Comparison for Calibrated Submanifolds.}

If a Riemannian manifold $M$ has an upper bound $K$ on its sectional curvatures then the volume of a sufficiently small ball in $M$ is greater then the volume of a ball of the same radius in a space form of constant curvature $K$. It turns out that this holds more generally for calibrated submanifolds of $M$ :

Theorem 2.0.1. Let $\eta$ be a calibrating $k$-form on a Riemannian manifold $M$ and $L$ be a calibrated submanifold. Let the sectional curvatures of $M$ be 
bounded from above by $K$. Let $r \leq \min \left(\operatorname{injrad}(M), \frac{\pi}{\sqrt{K}}\right)$. Let $p \in L, B(p, r)$ be a ball of radius $r$ around $p$ in $M$ and $B^{K}(r)$ be a ball of radius $r$ in $a$ $k$-dimensional space of constant sectional curvature $K$. Then

$$
\operatorname{vol}(L \bigcap B(p, r)) \geq \operatorname{vol}\left(B^{K}(r)\right) \text {. }
$$

Proof. The proof is based on the following lemma, which is a counterpart to Rauch comparison theorem:

Lemma 2.0.1. Let $M$ be a (complete) Riemannian manifold whose sectional curvature is bounded from above by $K$ and $\gamma:[0, t] \mapsto M$ be a unit speed geodesic. Let $Y$ be a Jacobi field along $\gamma$ which vanishes at 0 , orthogonal to $\gamma^{\prime}$ and assume that $t \leq \frac{\pi}{\sqrt{K}}$. Then its length $|Y(\theta)|$ satisfies the following differential inequality $|Y|^{\prime \prime}+K|Y| \geq 0$. Moreover if a function $\Psi$ is a solution to $\Psi^{\prime \prime}+K \cdot \Psi=0, \Psi(0)=0$ and $\Psi(t)=|Y(t)|$ then $\Psi(\theta) \geq|Y(\theta)|$ for $0 \leq \theta \leq t$.

Proof. First the condition on $t$ means that $Y$ doesn't vanish on $(0, t]$ by Rauch Comparison theorem. We have $|Y|=\sqrt{\langle Y, Y\rangle},|Y|^{\prime}=\frac{\left\langle\nabla_{t} Y, Y\right\rangle}{|Y|}$,

$$
\begin{aligned}
|Y|^{\prime \prime} & =\frac{\left|\nabla_{t} Y\right|^{2}-\left\langle Y, R\left(\gamma^{\prime}, Y\right) \gamma^{\prime}\right\rangle}{|Y|}-\frac{\left\langle\nabla_{t} Y, Y\right\rangle^{2}}{|Y|^{3}} \\
& \geq \frac{\left|\nabla_{t} Y\right|^{2}|Y|^{2}-\left\langle\nabla_{t} Y, Y\right\rangle^{2}}{|Y|^{3}}-K|Y| \geq-K|Y|
\end{aligned}
$$

by the Cauchy-Schwartz inequality. Here $R$ is the curvature operator, $\gamma^{\prime}$ is the (unit length) tangent field to $\gamma$. Since $Y$ is orthogonal to $\gamma^{\prime}$ then $\frac{\left\langle R\left(\gamma^{\prime}, Y\right) \gamma^{\prime}, Y\right\rangle}{|Y|^{2}}$ is the sectional curvature of the plane through $Y$ and $\gamma^{\prime}$, which is less then $K$.

For the second claim consider $F=\frac{|Y|}{\Psi} . \Psi$ is positive on the interval $(0, t]$ and hence $F$ is well defined on that interval. Also $F^{\prime}=\frac{|Y|^{\prime} \Psi-\Psi^{\prime}|Y|}{\Psi^{2}}$.

Consider $G=|Y|^{\prime} \Psi-\Psi^{\prime}|Y| . G(0)=0, G^{\prime}=|Y|^{\prime \prime} \Psi-\Psi^{\prime \prime}|Y| \geq 0$.

So $G \geq 0$, i.e., $F^{\prime} \geq 0$. Now $F(t)=1$, so $F \leq 1$ i.e., $|Y| \leq F$.

Now we can prove Theorem 2.0.1: Let $d_{p}$ be a distance function to $p$ on $M$. Then for an open dense set of full measure of values $t, t$ is a regular value of $d_{p}$ restricted to $L$. Let now

$$
f(t)=\operatorname{vol}(L \bigcap B(p, t)) \text { and } g(t)=\int_{L \bigcap B(p, t)}\left|\nabla_{L} d_{p}\right| .
$$


We can similarly define $\bar{f}(t)$ and $\bar{g}(t)$ on $\bar{L}$ - the $k$-dimensional space form of constant curvature $K$. Then $\bar{f}=\bar{g}$ because $\left|\nabla \overline{d_{p}}\right|=1$ on $\bar{L}$.

For $t$ a regular value as above we have by the co-area formula:

$$
f^{\prime}(t) \geq g^{\prime}(t), g^{\prime}(t)=\operatorname{vol}\left(S_{t}\right), \bar{f}^{\prime}=\operatorname{vol}\left(\overline{S_{t}}\right)
$$

here $S_{t}=d_{p}^{-1}(t) \bigcap L$ and $\overline{S_{t}}$ in the analogous object on $\bar{L}$.

Consider now a map $\xi: S_{t} \times[0, t] \mapsto M, \xi(a, \theta)=\exp \left(\frac{\theta}{t} \exp ^{-1}(a)\right)$. Here $a \in S_{t}, \theta \in[0, t]$. We claim that $\operatorname{vol}\left(\xi\left(S_{t} \times[0, t]\right)\right) \geq f(t)$. Indeed let $\rho$ be a $(k-1)$-form on $B(p, r)$ s.t. $d \rho=\eta$. By the calibrating condition:

$$
\operatorname{vol}\left(\xi\left(S_{t} \times[0, t]\right)\right) \geq \int_{S_{t} \times[0, t]} \xi^{*} \eta=\int_{S_{t}} \rho=\int_{B(p, t) \cap L} \eta=f(t) .
$$

Also on $\bar{L}$ we have $\overline{f(t)}=\operatorname{vol}\left(\bar{\xi}\left(\overline{S_{t}} \times[0, t]\right)\right)$. We need to estimate $h(t)=$ $\operatorname{vol}\left(\xi\left(S_{t} \times[0, t]\right)\right)$. Let $g^{\prime}$ be the product metric on $S_{t} \times[0, t]$. Then $h(t)=$ $\int_{S_{t} \times[0, t]} J a c(d \xi) d g^{\prime}$. Here $J a c(d \xi)$ is the Jacobian of $\xi$. To estimate $J a c(d \xi)$ at a point $(a, \theta) \in\left(S_{t},[0, t]\right)$ we take an o.n. basis $v_{1} \ldots v_{k-1}$ to $S_{t}$ at $a$. Then $d \xi\left(v_{i}\right)(a, \theta)$ is a value of a Jacobi field along a unit speed geodesic $\left(\exp \left(s \cdot \frac{\exp ^{-1}(a)}{t}\right) \mid s \in[0, \infty)\right)$ at $s=\theta$ which is orthogonal to this geodesic, vanishes at 0 and those length is 1 at $s=t$. Let $F_{t}(\theta)$ solve $F_{t}^{\prime \prime}+K \cdot F_{t}=0$, $F_{t}(0)=0, F_{t}(t)=1$. By Lemma 2.0.1 we have $\left|d \xi\left(v_{i}\right)(a, \theta)\right| \leq F_{t}(\theta)$, so

$$
J a c(d \xi)(a, \theta) \leq\left(F_{t}(\theta)\right)^{k-1} .
$$

We can consider an analogous situation on $\bar{L}$ and in that case we have an equality $\operatorname{Jac}(d \bar{\xi})=\left(F_{t}(\theta)\right)^{k-1}$. So

$$
\begin{aligned}
\overline{f(t)}=\int_{\overline{S_{t}} \times[0, t]}\left(F_{t}(\theta)\right)^{k-1} & =\operatorname{vol}\left(\overline{S_{t}}\right) \cdot \int_{[0, t]}\left(F_{t}(\theta)\right)^{k-1} d \theta \\
& =(\text { by }(1))=\bar{f}^{\prime}(t) \cdot \alpha(t) .
\end{aligned}
$$

Here $\alpha(t)=\int_{[0, t]}\left(F_{t}(\theta)\right)^{k-1} d \theta$. Returning now to our calibrated submanifold we deduce from $(2)$ and $(1)$ that $f(t) \leq f^{\prime}(t) \cdot \alpha(t)$. So $\frac{f^{\prime}(t)}{f(t)} \geq \frac{\bar{f}^{\prime}(t)}{\bar{f}(t)}$, i.e., $\ln (f)^{\prime} \geq(\ln (\bar{f})-\epsilon)^{\prime}$ for any $\epsilon>0$. Having $\epsilon$ fixed we can choose $t_{0}$ small enough s.t. $\ln f\left(t_{0}\right) \geq \ln \bar{f}\left(t_{0}\right)-\epsilon$.

Now $\ln f(\theta)$ is defined for a.e. $\theta$ and is an increasing function of $\theta$, so

$$
\ln f(t) \geq \ln f\left(t_{0}\right)+\int_{\left[t_{0}, t\right]} \ln f^{\prime} \geq \ln \bar{f}\left(t_{0}\right)-\epsilon+\int_{\left[t_{0}, t\right]}(\ln \bar{f})^{\prime}=\ln \bar{f}(t)-\epsilon
$$


Now $\epsilon$ was arbitrary, hence $\ln f(t) \geq \ln \bar{f}(t)$ i.e. $f(t) \geq \bar{f}(t)$.

We now come to the main result of this section. We wish to strengthen Theorem 2.0.1 by the analogous result for volumes of balls of radius $r$ in the induced metric on calibrated submanifolds (which are smaller then the balls we considered before). We have the following:

Theorem 2.0.2. Let $M, \eta, p, L, K$ and $B^{K}(r)$ be as in Theorem 2.0.1 and let $d_{L}$ be the distance function to $p$ on $L$ in the induced metric on $L$. Then for $r \leq \min (\operatorname{injrad}(M), R(K))$ we have:

$$
\operatorname{vol}\left(x \in L \mid d_{L}(x) \leq r\right) \geq \operatorname{vol}\left(B^{K}(r)\right) .
$$

Here $R(K)>0$ and $R(K)=\pi / \sqrt{K}$ for $K$ positive.

Corollary 2.0.1. Let $M, \eta$ be as before. Then there is an a priori bound on a diameter of submanifolds of $M$ calibrated by $\eta$ in a given homology class $\beta \in H_{k}(M, \mathbb{Z})$.

Proof. Choose some $r$ satisfying the conditions of Theorem 2.0.2. Let $L$ be some calibrated submanifold representing the homology class $\beta$. Let $\Gamma$ be a maximal covering of $L$ by disjoint balls of radius $r$. By Theorem 2.0.2 each such ball has volume at least $\epsilon=\epsilon(r)$ and the volume of $L$ is $v=[\eta](\beta)$. So the number of elements in $\Gamma$ is at most $N=\frac{v}{\epsilon}$. Every point in $L$ is contained in one of the balls of radius $2 r$ with the same centers as the balls in $\Gamma$. From this it is easy to deduce that the diameter of $L$ is at most $4 r N$.

Proof of Theorem 2.0.2. We wish to use the same argument as in the proof of Theorem 2.0.1 for the distance function $d_{L}$. The problem is that $d_{L}$ is not a smooth function in the $r$-neighbourhood of $p$. But we can still smoothen it using the following technical lemma:

Lemma 2.0.2. Let $L \subset M$ be a submanifold, $p \in L$ and $d_{L}$ as before. We can pick $\rho>0$ and $a\left(C^{\infty}\right)$ function $0 \leq \nu \leq 1$ on $[0, \infty)$ which is 0 on $(0, \rho], 1$ on $[2 \rho, \infty)$ and nondecreasing s.t. for any $\epsilon>0$ there is a function $\lambda_{\epsilon}$ on $L$ which satisfies:

1) $\lambda_{\epsilon}$ is $C^{\infty}$ outside of $p$,

2) $d_{L} \leq \lambda_{\epsilon} \leq d_{L}(1+\epsilon)$, 
3) $\left|\nabla \lambda_{\epsilon}\right| \leq 1+\nu\left(d_{L}\right) \epsilon$.

Proof. Pick a positive $\rho \ll \operatorname{injrad}(L)$. Choose a function $\kappa$ on $M$ s.t. $\kappa=1$ on $B(p, 2 \rho)$ and $\kappa=0$ outside of $B(p, 3 \rho)$. Choose a nonnegative radially symmetric function $\sigma$ on $\mathbb{R}^{k}$ with support in the unit ball which integrates to 1 and let $\sigma_{n}(x)=n^{k} \cdot \sigma(n x)$. Then $\sigma_{n}$ also integrates to 1 .

Choose a nonnegative function $\phi \leq 1, \phi=0$ on $B\left(p, \frac{5 \rho}{4}\right)$ and $\phi=1$ outside of $B\left(p, \frac{3 \rho}{2}\right)$. For each integer $n$ we define a function $\mu^{n}$ on $L$,

$$
\mu^{n}(q)=\int_{T_{q} L} d_{L}\left(\exp _{L}(\theta)\right) \sigma^{n}(\theta) d \theta .
$$

Here $T_{q} L$ is the tangent space to $L$ at $q$. Since $\sigma^{n}$ was radially symmetric function and $T_{q} L$ has a metric, the expression $\sigma^{n}(\theta)$ is well defined and also the integration takes part only on a ball of radius $\frac{1}{n} \subset T_{q} L$.

Also it is clear that

$$
\mu^{n}=d_{L}+o\left(\frac{1}{n}\right)
$$

The point is that for large $n, \mu^{n}$ is a smooth function on $L$. Indeed let us denote by $J(a, b)$ the Jacobian of the exponential map on $L$ from $T_{a} L$ that hits $b$ for $a, b$ points in $L$ that are close enough. Then $J(a, b)$ is a smooth function of $(a, b)$ and we can rewrite

$$
\mu^{n}(q)=\int_{L} J(q, b)^{-1} \cdot d_{L}(b) \cdot \sigma\left(\exp _{b}^{-1}(q)\right) d b .
$$

It is clear from this definition that $\mu^{n}$ is a smooth function of $q$ for $n$ large enough. Also one can prove that $\left|\mu^{n}\left(q_{1}\right)-\mu^{n}\left(q_{2}\right)\right| \leq d\left(q_{1}, q_{2}\right) \cdot\left(1+o\left(\frac{1}{n}\right)\right)$, so

$$
\left|\nabla \mu^{n}\right| \leq 1+o\left(\frac{1}{n}\right) \text {. }
$$

Now pick $\epsilon>0$. Define $\lambda_{\epsilon}^{n}=(1+\phi \epsilon)\left(\kappa \cdot d_{L}+(1-\kappa) \cdot \mu^{n}\right)$. Then $\lambda_{\epsilon}^{n}=d_{L}$ on $B\left(p, \frac{3 \rho}{2}\right)$ and it is smooth outside of $p$. One can also directly verify that we can choose a constant C s.t. for sufficiently large $n$, the function $\lambda_{\epsilon}=\lambda_{\frac{\epsilon}{C}}^{n}$ satisfies properties 2) and 3) as desired.

Now we can prove Theorem 2.0.2: We will use the fact that the function $\alpha(t)$, defined in the proof of Theorem 2.0.1, is an increasing function of $t$ for $0 \leq t \leq \frac{\pi}{\sqrt{K}}$ for $K$ positive and for $0 \leq t \leq R(K)$ for $K$ negative. 
Pick $\rho$ as in Lemma 2.0.2. Let $\epsilon>0$. We will follow the lines of proof of Theorem 2.0.1 for the function $\lambda_{\epsilon}$ instead of the distance function. We denote by

$$
f(t)=\operatorname{vol}\left(\lambda_{\epsilon}^{-1}([0, t]), S_{t}=\lambda_{\epsilon}^{-1}(t) .\right.
$$

Then conditions on $\lambda_{\epsilon}$ and the co-area formula imply that for a regular value $t$ we have $f^{\prime}(t) \geq \frac{\operatorname{vol}\left(S_{t}\right)}{1+\epsilon(t)}$.

Also we can consider $A_{t}=\left((a, \theta) \mid a \in S_{t}, 0 \leq \theta \leq d_{p}(a)\right)$ (here $d_{p}$ is the distance to $p$ in the ambient manifold). We have $\xi: A_{t} \mapsto M, \xi(a, \theta)=$ $\exp _{M}\left(\frac{\theta \cdot \exp ^{-1}(a)}{d_{p}(a)}\right)$.

As before we will have $f(t) \leq \operatorname{vol}\left(\xi\left(A_{t}\right)\right)$ and $J a c(d \xi) \leq\left(F_{d_{p}(a)}(\theta)\right)^{k-1}$ (see (2), we have the same notations as in Theorem 2.0.1). The estimate for Jacobian is true for the following reason: Let $v_{1}, \ldots, v_{k-1}$ be an o.n. basis to $S_{t}$ at $a$. Then only the normal component of $d \xi\left(v_{i}\right)$ to the geodesic contributes to $J a c(d \xi)$. The length of the normal component can be estimated by Lemma 2.0.1.

So we will have $\operatorname{vol}\left(\xi\left(A_{t}\right)\right) \leq \int_{S_{t}} \alpha\left(d_{p}(a)\right) d a \leq \operatorname{vol}\left(S_{t}\right) \cdot \alpha(t)$ (here we used the fact that $\alpha$ is an increasing function and $\left.d_{p}(a) \leq d_{L}(a) \leq \lambda_{\epsilon}(a)=t\right)$.

Combining all this we get

$$
(\ln f)^{\prime}(t) \geq \frac{(\ln \bar{f})^{\prime}(t)}{1+\epsilon \nu(t)}=\left[\left(\frac{\ln \bar{f}}{1+\epsilon \nu}\right)^{\prime}+\epsilon \nu^{\prime} /(1+\epsilon \nu)^{2} \cdot \ln (\bar{f})\right](t) .
$$

Now $\nu(t)=0$ for $t \leq \rho$ and $\nu^{\prime}(t)=0$ for $t \geq 2 \rho$ and $\ln (\bar{f}) \geq-C$ for $2 \rho \geq t \geq \rho$. Here $C$ depends on $\rho$, but not on $\epsilon$. So

$$
(\ln f)^{\prime} \geq\left(\frac{\ln \bar{f}}{1+\epsilon \nu}\right)^{\prime}-\epsilon C^{\prime}
$$

i.e., $\left(\ln f+\epsilon C^{\prime} t\right)^{\prime} \geq\left(\frac{\ln \bar{f}}{1+\underline{\epsilon \nu}}\right)^{\prime}$. Also for $\theta$ small we have $f(\theta)=\theta^{k}\left(1+o\left(\theta^{2}\right)\right)$ and the same holds for $\bar{f}$ (here $k$ is the dimension of $L$ ). So we have

$$
\ln (f(\theta))+\epsilon C^{\prime} \theta \geq \ln (\bar{f}(\theta))=\frac{\ln \bar{f}(\theta)}{1+\epsilon \nu(\theta)} .
$$

From all this we deduce that $: \ln (f)+\epsilon C^{\prime} t \geq \ln \bar{f} /(1+\epsilon \nu)$. Here $\epsilon$ was arbitrary and we are done. 


\section{Special Lagrangian geometry on almost Calabi-Yau manifolds.}

\subsection{Basic properties.}

Let $\left(M^{2 n}, \omega, \varphi\right)$ be an almost Calabi-Yau manifold (see the Introduction). In this section we will explore some basic facts regarding the moduli-space of SLag submanifolds on $M$.

Let $L$ be a SLag submanifold of $M$. If $\omega$ is a Calabi-Yau metric, then R.C. McLean proved in Corollary 3.9 of [6] that the moduli-space of SLag submanifolds containing $L$ is smooth of dimension $b_{1}(L)$. We will now demonstrate that the same holds for any Kahler form $\omega$ on $M$ :

Lemma 3.1.1. Let $L^{n}$ be a compact connected $n$-dimensional manifold. Then the moduli-space of SLag embeddings of $L$ into $M$ is a smooth (though not necessarily a paracompact) manifold of dimension $b_{1}(L)$.

Proof. The proof is a slight modification of McLean's proof for a Calabi-Yau metric (see [6], Corollary 3.9).

Let $i: L \mapsto M$ be a (smooth) SLag embedding of $L$ into $M$. Locally the moduli-space $\Gamma$ of $C^{2, \alpha}$-embeddings of $L$ into $M$ (modulo the diffeomorphisms of $L$ ) can be identified with the $C^{2, \alpha}$ sections of the normal bundle of $i(L)$ to $M$ via the exponential map. Also the normal bundle is naturally isomorphic to the cotangent bundle of $L$ via the map $\left.v \mapsto i_{v} \omega\right|_{L}$. Hence the tangent space to $\Gamma$ at $L$ can be identified with $C^{2, \alpha} 1$-forms on $L$. Let $V_{k}$ be the vector space of exact $C^{1, \alpha} k$-forms on $L$ and let $V=V_{2}^{\prime} \oplus V_{n}$. There is locally a map $\sigma: \Gamma \mapsto V$ given at an embedding $j(L) \in \Gamma$ by

$$
\left(j^{*}(\omega), j^{*}(\operatorname{Im} \varphi)\right)
$$

The moduli-space $\Phi$ of SLag embeddings is just the zero set of $\sigma$. The differential of $\sigma$ at $i(L)$ in the direction of $\alpha$ (where $\alpha$ is a $C^{2, \alpha} 1$-form on $\mathrm{L}$ as above) is

$$
(d \alpha, d(f * \alpha))
$$

where $f$ is the length of $\varphi$ in the metric defined by $\omega$. We claim that the differential $d \sigma$ is surjective and the tangent space to $\Phi$ is naturally isomorphic to the first cohomology $H^{1}(L, \mathbb{R})$. To prove this consider first an operator $P$ from the space of $C^{3, \alpha}$ functions on $L$ to the space of $C^{1, \alpha}$ $n$-forms on $L, P(h)=d(f * d h)$. We claim that $P$ is surjective onto the space 
$V_{n}$ of exact $n$-forms and and the kernel of $P$ is the space of constant functions on $L$. Since $f$ is non-vanishing $P$ is elliptic. To prove the surjectivity of $P$ onto $V_{n}$ it is enough to show that the co-kernel of $P$ consists of constant multiples of the volume form on $L$. Let $\mu$ be in the co-kernel of $P$. Let $h=* \mu$. One easily computes that

$$
\int_{L} P h \cdot \mu= \pm \int_{L} f\left|d^{*}(\mu)\right|^{2} .
$$

So $d^{*}(\mu)=0$, hence $\mu$ is a constant multiple of the volume form on $M$. Let now $h$ be in the kernel of $P$. Then arguing as before we get that $\mu=* h$ is a constant multiple of the volume form on $M$, i.e., $h$ is a constant.

Now we can prove the lemma. First we prove that $d \sigma$ is surjective. Let $\alpha$ be an exact 2 -form on $L$, and $\beta$ be an exact $n$-form on $L$. We need to find a 1 -form $\gamma$ on $L$ s.t.

$$
d \gamma=\alpha, d(f * \gamma)=\beta .
$$

Since $\alpha$ is exact there is a 1-form $\gamma^{\prime}$ s.t. $d \gamma^{\prime}=\alpha$. We are looking for $\gamma$ of the form $\gamma=\gamma^{\prime}+d h$ for a function $h$. Since the operator $P$ is surjective onto $V_{n}$, we get that such $h$ exists, so $d \sigma$ is surjective, hence $\Phi$ is smooth. Next we prove that $\operatorname{dim}(\Phi)=b_{1}(L)$. Let $W=\operatorname{ker}(d \sigma)$. $W$ is the tangent space to $\Phi$ at $i(L)$. Since $W$ is represented by closed 1-forms on $L$, there is a natural $\operatorname{map} \xi: W \mapsto H^{1}(L, \mathbb{R})$. We claim that this map is an isomorphism. Indeed let $a \in H^{1}(L, \mathbb{R})$ and let $\gamma^{\prime}$ be a closed 1-form on $L$ representing the class $a$. From the properties of the operator $P$ it is clear that there is a unique exact 1-form $\gamma^{\prime \prime}=d h$ s.t. $\gamma=\gamma^{\prime}+\gamma^{\prime \prime}$ is in the kernel of $\sigma$. Hence $\xi$ is an isomorphism.

Remark 1. For a more general setup of deformations of SLag submanifolds in symplectic manifolds with trivialized canonical bundle we refer the reader to $[10]$.

Let $i: L \mapsto M$ be a SLag submanifold in $M$. By the moduli-space $\Phi$ of SLag submanifolds through $L$ we mean a connected component of the space of SLag embeddings of $L$ into $M$ which contains the embedding $i(L)$. Next we investigate the connections between $\Phi$ and the automorphisms of $M$.

Lemma 3.1.2. Let $g$ be an automorphism of $(M, \omega, \varphi), L$ be a SLag submanifold of $M$ invariant under $g$ and suppose $g$ acts trivially on the first cohomology of $L$. Then $g$ leaves invariant every element in the moduli-space $\Phi$ of SLag submanifolds through $L$. Moreover suppose that $x \in M-L$ in an isolated fixed point of $g$. Then $x$ cannot be contained in any element of $\Phi$. 
Proof. Since $g$ is structure preserving, it sends SLag submanifolds to SLag submanifolds. Since $g$ leaves $L$ invariant, it preserves $\Phi$. From the identification of the tangent space of $\Phi$ at $L$ with $H^{1}(L, \mathbb{R})$ and the fact that $g$ acts trivially on $H^{1}(L, \mathbb{R})$ we deduce that $g$ acts trivially on the tangent space to $\Phi$ at $L$. Hence $g$ acts trivially on $\Phi$, i.e., it leaves each element of $\Phi$ invariant.

To prove the second statement, consider the set $S$ of those elements in $\Phi$ which contain $x$. Obviously $S$ is closed and doesn't contain $L$. We prove that $S$ is open and then it will be empty.

Let $L^{\prime} \in S$. Let $L^{\prime \prime} \in \Phi$ be close to $L^{\prime}$. Then $L^{\prime \prime}$ can be viewed uniquely as an image $\exp (v)\left(L^{\prime}\right)$, where $v$ is a normal vector field to $L^{\prime}$. Suppose $v(x) \neq 0$. Since $L^{\prime \prime}$ is $g$-invariant then $\exp (d g(v(x)))$ is also in $L^{\prime \prime}$, where $d g$ is the differential of $g$ at $x$. Since $L^{\prime}$ is $g$-invariant then $d g$ preserves the tangent space to $L^{\prime}$ at $x$, hence it preserves the normal space to $L^{\prime}$ at $x$. Also since $x$ is an isolated fixed point then $d g$ has no nonzero invariant vectors. Hence $v(x) \neq d g(v(x))$.

Since exponential map is a diffeomorphism from a small neighbourhood of the normal bundle of $L^{\prime}$ to $M$ we see that $\exp (d g(v(x)))$ is not in $L^{\prime \prime}$ - a contradiction. So $v(x)=0$ i.e., $L^{\prime \prime} \in S$.

Lemma 3.1.2 can be used to show that in some cases the moduli-space $\Phi$ of SLag submanifolds through $L$ is not compact. Suppose that $b_{1}(L)=n$. We say that $L$ satisfies the condition $\star$ if for $\alpha_{1}, \ldots, \alpha_{n}$ a basis for $H^{1}(L)$ we have $\alpha_{1} \cup \ldots \cup \alpha_{n} \neq 0$. This holds e.g., if $L$ is a torus.

Lemma 3.1.3. Let $L$ be a SLag submanifold of $M$ with $b_{1}(L)=n$ s.t. $L$ satisfies the condition $\star$. Suppose we have $g, x$ satisfying the conditions of Lemma 3.1.2. Then $\Phi$ is not compact.

Proof. We have a fiber bundle $F$ over $\Phi$,

$$
F \subset M \times \Phi, F=\left(\left(a, L^{\prime}\right) \mid L^{\prime} \in \Phi, a \in L^{\prime}\right)
$$

( $F$ is the total space of $\Phi$ ). We have a projection map $p r: F \mapsto \Phi$ onto the second factor (which is a fibration) and the evaluation map $e v: F \mapsto M$, $e v\left(a, L^{\prime}\right)=a$. The tangent space to $F$ at a point $\left(a, L^{\prime}\right) \in F$ naturally splits as

$$
T_{\left(L^{\prime}, a\right)} \Phi=T_{a} L^{\prime} \oplus T^{\prime}
$$


Here $T_{a} L^{\prime}$ is the tangent space to $L^{\prime}$ at $a$ (the tangent space to the fiber of the projection $p r)$ and $T^{\prime}=((v(a), v) \mid v$ is a normal variation vector field in $T_{L} \Phi$ and $a(v)$ is the value of $v$ at $\left.a\right)$.

We note that $\Phi$ is orientable, in fact it has a natural volume element $\sigma$. Let $L^{\prime} \in \Phi$ and $v_{1}, \ldots, v_{n}$ be elements of the tangent space to $\Phi$ at $L^{\prime}$. According to the proof of Lemma 3.1.1 we can view $v_{1}, \ldots, v_{k}$ as elements of $H^{1}\left(L^{\prime}, \mathbb{R}\right)$. We define:

$$
\sigma\left(v_{1}, \ldots, v_{k}\right)=v_{1} \cup \ldots \cup v_{k}\left(L^{\prime}\right) .
$$

$F$ is also orientable, in fact the $2 n$-form $\alpha=p r^{*}(\sigma) \wedge e v^{*}(\operatorname{Re} \varphi)$ is a volume form on $F$. Suppose that $\Phi$ is compact, then so is $F$. We will prove that the degree of the evaluation map ev $: F \mapsto M$ is positive. Let $\eta$ be the Riemannian volume form on $M$. Then

$$
\operatorname{deg}(e v)=\int_{F} e v^{*}(\eta) / \operatorname{vol}(M) .
$$

Let $L_{\phi} \in \Phi$. We can view $L_{\phi} \subset F$ as a fiber of the projection $p r: F \mapsto \Phi$. Let $\alpha_{1}, \ldots, \alpha_{n}$ be a basis for $H^{1}\left(L_{\phi}\right)$ s.t. $\alpha_{1} \cup \ldots \cup \alpha_{n}\left[L_{\phi}\right]=1$. Then $\left(\alpha_{i}\right)$ give rise to the corresponding vector fields $v_{1}, \ldots, v_{n}$ along $L_{\phi} \subset F$, which form a frame for the bundle $T^{\prime}$ (see (3)) along $L_{\phi}$. So $i_{v_{j}} e v^{*}(\omega)$ restricted to $L_{\phi}$ represents the cohomology class $\alpha_{j}$ on $L_{\phi}$ and $p r^{*}(\sigma)\left(v_{1}, \ldots, v_{n}\right)=1$. Since $p r: F \mapsto \Phi$ is a fiber bundle we can use the integration over the fiber formula to compute:

$$
\int_{F} e v^{*}(\eta)=\int_{\Phi}\left(\int_{L_{\phi}} i_{v_{1}} \ldots i_{v_{n}} e v^{*}(\eta)\right) d \phi
$$

Also $i_{v_{1}} \ldots i_{v_{n}} e v^{*}(\eta)$ is easily seen to be equal to

$$
i_{v_{1}} e v^{*}(\omega) \wedge \ldots \wedge i_{v_{n}} e v^{*}(\omega)
$$

(all restricted to the fiber $L_{\phi}$ ). So

$$
\int_{L_{\phi}} i_{v_{1}} \ldots i_{v_{n}} e v^{*}(\eta)=\alpha_{1} \cup \ldots \cup \alpha_{n}\left[L_{\phi}\right]=1 .
$$

So $\operatorname{deg}(e v)=\int_{\Phi} 1 / \operatorname{vol}(M)=\operatorname{vol}(\Phi) / \operatorname{vol}(M)>0$.

Suppose $g, x$ satisfy the conditions of Lemma 3.1.2. Since $\operatorname{deg}(e v)>0$, $e v$ is surjective. But $x$ is not in the image of $e v-$ a contradiction.

Next we would like to discuss a connection between automorphisms of $(M, \omega, \varphi)$ and SLag fibrations on $M$ (see Definition 1.0.1): 
Lemma 3.1.4. Let $M$ be an almost Calabi-Yau manifold with a SLag torus fibration $\alpha: M \mapsto S$ and a (non-trivial) automorphism $g$ of $M$, which commutes with $\alpha$. Then the fixed point set fix $(g)$ is contained in $M-M_{0}$. Suppose moreover that $S$ is a smooth manifold and $\alpha$ is a smooth map. Then for any point $p \in f i x(g)$ the differential $d \alpha$ of $\alpha$ is not surjective at $p$ (i.e., fix $(g)$ is contained in the singular locus of $\alpha$ ).

Proof. Suppose that $m$ is a point in $f i x(g) \cap M_{0}$. Let $L$ be the fiber of $\alpha$ through $m$ and let $N_{m}$ be the normal bundle of $L$ in $M$ at $m$. Then $N_{m}$ is a Lagrangian subspace of $T_{m} M$. Also $g$ preserves $L$, hence the differential $d g$ of $g$ at $m$ acts on $N_{m}$. We claim that this action can't be trivial. Indeed $d g$ is complex-linear on $T_{m} M$, so if the $d g$-action is trivial on $N_{m}$, it would be trivial on the complexification $N_{m}^{c}$ of $N_{m}$ in $T_{m} M$. But $N_{m}^{c}=T_{m} M$, and so the $g$-action would be trivial on $M$ - a contradiction.

So there is a vector $0 \neq v \in N_{m}$ s.t. $d g(v) \neq v$. For any $t \in \mathbb{R}$ consider the level set $L_{t}$ of $\alpha$ passing through $\exp (t v)$. Then $L_{t}$ is $g$-invariant, hence the point $\exp (t d g(v))$ is also contained in $L_{t}$. But level sets of $\alpha$ near $L$ are given uniquely as graphs of normal vector fields on $L$ via the exponential map. Hence $\exp (t d g(v))$ cannot be contained on $L_{t}$ - a contradiction.

Suppose now that $S$ is smooth and $\alpha$ is a smooth map. Let $p \in f i x(g)$ and assume that $d \alpha$ is surjective at $p$. The level set $L$ of $\alpha$ through $p$ is a smooth Lagrangian submanifold of $M$ near $p$. Arguing as before we get to a contradiction.

Remark 2. The fixed point set of a (non-trivial) automorphism of an almost Calabi-Yau manifold has complex codimension at least 2, i.e. real codimension at least 4. Moreover this codimension is attained in many examples. Also the singular locus of a hypothetical SLag torus fibration has real codimension 4 . Thus the previous lemma potentially suggests the location of the singular locus of a SLag torus fibration on an almost CalabiYau manifold.

\subsection{SLag torus fibration on a Borcea-Voisin threefold.}

In this section we investigate one example of an almost Calabi-Yau manifold in detail- the Borcea-Voisin threefold $M$. It will turn out that for a suitable choice of a Kahler metric on $M$ we can prove that $M$ has a SLag torus fibration.

The property, which we will utilize in studying $M$, is the fact that $M$ 
is a union of neighbourhoods, each of those is biholomorphic to a product of an elliptic curve $E$ with an open subset $\bar{X}$ of a $K 3$ surface. We will define a certain Kahler metric on $M$ and a moduli-space $\Phi$ of SLag tori on $M$ such that in any such neighbourhood the elements of $\Phi$ will look like $S^{1} \times T$, where $S^{1}$ is a circle in $E$ and $T$ is a pseudoholomorphic 2-torus for an appropriate almost complex structure on $\bar{X}$. We will use Gromov's compactness theorem to study the compactification $\bar{\Phi}$ of our moduli-space $\Phi$ of SLag tori. A crucial point will be the fact that the boundary $\partial \Phi=\bar{\Phi}-\Phi$ has dimension 1 (i.e. co-dimension 2). We will show that the image $e v(F)$ of the total space $F$ of $\Phi$ under the evaluation map (see section 3.1) is open in $M$. Moreover we will use $\partial \Phi$ to show that the boundary of $e v(F)$ in $M$ is contained in the image $i: N \mapsto M$ of a smooth map $i$ from a 4-dimensional compact manifold $N$ into $M$. This proves that the total space of $\bar{\Phi}$ fills the whole $M$. Moreover we will see that different elements of $\bar{\Phi}$ don't intersect in $M$ and $M$ has a SLag fibration $\alpha$ over $\bar{\Phi}$ according to Definition 1.0.1.

We adopt the definition of $M$ from [5], so we define $M$ to be the resolution of singularities of a 6 -torus $T^{6}=T^{2} \times T^{2} \times T^{2}$ by a $\mathbb{Z}_{2} \oplus \mathbb{Z}_{2}$-action, where the generators of the $\mathbb{Z}_{2}$ actions are:

$$
\begin{gathered}
\alpha: z_{1} \rightarrow-z_{1}+\frac{1}{2}, z_{2} \rightarrow-z_{2}+\frac{1}{2}, z_{3} \rightarrow z_{3} \\
\beta: z_{1} \rightarrow-z_{1}, z_{2} \rightarrow z_{2}, z_{3} \rightarrow-z_{3} .
\end{gathered}
$$

The fixed locus of $\alpha$ is 16 2-tori : $A \times A \times T^{2}$ and the one of $\beta$ is 16 2-tori: $B \times T^{2} \times B$.

Here $A \subset T^{2}$ is a set $\left\{\frac{2+2 i \pm 1 \pm i}{4}\right\}$ and $B \subset T^{2}$ is a set $\left\{0, \frac{1}{2}, \frac{i}{2}, \frac{i+1}{2}\right\}$. The fixed loci of $\alpha$ and $\beta$ do not intersect. Also $\alpha \circ \beta$ has no fixed points.

Consider a fixed torus $T^{2}$ (say of $\alpha$ ). Near $T^{2}$ the quotient looks like

$$
V=(U / \pm 1) \times T^{2} .
$$

Here $U$ is a ball of radius $r$ around a fixed point in $T^{4}$ (we can also view $U$ as a neighbourhood of the origin in $\mathbb{C}^{2}$ ). The resolution of singularities $U / \pm 1$ is a neighbourhood $\bar{U}$ of the zero set in the total space of $\gamma^{\otimes 2}$, where $\gamma$ is the universal line bundle over $\mathbb{C} P^{1}$ (thus the singular point will be replaced by $\left.\mathbb{C} P^{1}\right)$. We get a resolution of singularities of $T^{6} / Z_{2} \oplus Z_{2}$ by replacing each neighbourhood $V$ as in (4) by a neighbourhood

$$
\bar{V}=\bar{U} \times T^{2} \text {. }
$$

$\bar{U}$ has 1-parameter family of HyperKahler metrics $\omega_{t}$ (the Eguchi-Hanson metrics) (see [4], p. 304). Their Kahler potentials $f_{t}$ are given by

$$
f_{t}(u)=\sqrt{u^{2}+t^{2}}+t^{2} \log u-t^{2} \log \left(\sqrt{u^{2}+t^{2}}+t^{2}\right)
$$


here $u=\left|z_{1}\right|^{2}+\left|z_{2}\right|^{2}$. The Eguchi-Hanson Kahler form $\omega_{t}$ can be glued to the Euclidean metric outside of $\bar{U}$ by gluing their Kahler potentials for $t$ small enough (see [4]). Thus we obtain a Kahler form $\omega^{\prime}$ on $\bar{U}$. We consider the corresponding product metric in $\bar{V}$ (see (5)). Doing this for every fixed 2 -torus (both of $\alpha$ and of $\beta$ ) we get a Kahler metric on $M$, which is Euclidean outside of the neighbourhoods $\bar{V}$ as in (5). Also the holomorphic volume form $\varphi=i d z_{1} \wedge d z_{2} \wedge d z_{3}$ on $T^{6}$ lifts to a holomorphic volume form on $M$, thus $M$ becomes an almost Calabi-Yau manifold. Here is the main result of section 3.2 , whose proof will occupy the rest of section 3.2 :

Theorem 3.2.1. The Borcea-Voisin 3-fold $(M, \omega, \varphi)$ admits a SLag fibration according to Definition 1.0.1.

Proof. Consider a family of 3-tori $T_{a, b, c} \subset M$,

$$
T_{a, b, c}=\left(\left(z_{1}, z_{2}, z_{3}\right) \mid \operatorname{Re} z_{1}=a, \operatorname{Re} z_{2}=b, \operatorname{Re} z_{3}=c\right)
$$

which don't intersect the neighbourhoods $\bar{V}$ of fixed components as in (5). $T_{a, b, c}$ will be SLag tori in $M$. We would like to see what happens to this family then its elements intersect some neighbourhood $\bar{V}$ as in (5).

We wish to point out that P. Lu considered SLag submanifolds for a Calabi-Yau metric on $M$ in [5]. He was able to produce a big open set of those submanifolds. We are using a different metric and this will allow us to characterize the compactified moduli-space and to prove that $M$ fibers over it.

We return to the question of characterizing those elements which intersect a 'bad' neighbourhood $\bar{V}$. If this is a neighbourhood of a fixed component of $\alpha$, we consider the following setup: Let $\mathbb{Z}_{2}$ act on $T^{4}$ with a generator

$$
\alpha^{\prime}: z_{1} \rightarrow-z_{1}-\frac{1}{2}, z_{2} \rightarrow-z_{2}-\frac{1}{2} .
$$

This action has 16 fixed points and the resolution of singularities gives a $K 3$ surface. Also $M$ can be viewed as a resolution of singularities of

$$
\left(T^{4} / \alpha^{\prime} \times T^{2}\right) / \beta
$$

For each fixed point of $\alpha^{\prime}$ we introduce a neighbourhood $U \subset T^{4}$ as before (see (4)). We consider a bigger neighbourhood

$$
X=\left(\left(z_{1}+i a, z_{2}+i b\right) \mid\left(z_{1}, z_{2}\right) \in U, a, b \in \mathbb{R} / \mathbb{Z}\right)
$$


in $T^{4}$ and a corresponding neighbourhood $X^{\prime}$ in the quotient $T^{4} / \alpha^{\prime}$. We have a resolution of singularities $\bar{X}$ of $X^{\prime}$ (now we have 4 singular points in $\left.X^{\prime}\right)$ and a corresponding domain $\bar{W}=\bar{X} \times T^{2}$ in $M$ (since $\beta(\bar{W}) \cap \bar{W}=\emptyset$, we can view $\bar{W}$ as a domain in $M$ ). The moduli-space $T_{a, b, c}$ of SLag tori in $M$ as in (6) fills the complement of all those neighbourhoods $\bar{W}$ in $M$. We need to understand what happens to this moduli-space when its elements intersect such a neighbourhood $\bar{W}$.

Consider a canonical $(2,0)$-form $\eta=d z_{1} \wedge d z_{2}$ on $X \subset T^{4}$. The collection $w, R e \eta, I m \eta$ is the standard HyperKahler package on $T^{4} . \eta$ lifts to a holomorphic (2,0)-form on $\bar{X}$ and we have $i \cdot \eta \wedge d z_{3}=\varphi$ on $\bar{W}$. So the almost Calabi-Yau structure on $\bar{W}$ is a product structure. On $\bar{X}$ we have a moduli-space of embedded SLag tori with respect to $\left(\omega^{\prime}, \eta\right)$ (here $\omega^{\prime}$ is the Kahler form on $\bar{X}$ described earlier in this section). We have a connected component $\Phi^{\prime}$ of this moduli-space, which contains the SLag tori of the form

$$
T_{a, b}=\left(\left(z_{1}, z_{2}\right) \mid \operatorname{Re} z_{1}=a, \operatorname{Re} z_{2}=b\right) \subset \bar{X} .
$$

Those SLag tori give a fibration of the neighbourhood of the boundary of $\bar{X}$. By Lemma 3.1.1 $\Phi^{\prime}$ is a smooth 2-dimensional manifold. We can consider the space $\Phi^{\prime \prime}$ of submanifolds of $\bar{W}$ of the form

$$
\Phi^{\prime \prime}=\left(L^{\prime}=L \times T_{c} \mid L \in \Phi^{\prime}, T_{c}=(z \mid \operatorname{Re} z=c) \subset T^{2}\right) .
$$

The elements of $\Phi^{\prime \prime}$ are SLag tori in $\bar{W}$ and $\Phi^{\prime \prime}$ is a 3-dimensional manifold, contained in $\Phi$, which is also 3-dimensional. Next we investigate $\Phi^{\prime \prime}$ and show that $\Phi^{\prime \prime}$ is the set of those elements in $\Phi$, which intersect $\bar{W}$.

To investigate $\Phi^{\prime \prime}$ consider the almost Calabi-Yau manifold $\left(\bar{X}, \omega^{\prime}, \eta^{\prime}\right)$. We can conformally scale $\omega^{\prime}$ to some Hermitian 2 -form $\omega^{\prime \prime}$ on $\bar{X}$ s.t. in the metric $g^{\prime \prime}$ defined by $\omega^{\prime \prime}$ the form $\eta$ will have length 2 . So each of the 2 -forms $\omega, \operatorname{Re}, \operatorname{Im} \eta$ is a self-dual 2-form, which has length $\sqrt{2}$ with respect to $g^{\prime \prime}$. If $\mu$ is a self-dual 2-form of length $\sqrt{2}$ with respect to $g^{\prime \prime}$, then it induces an almost complex structure $I_{\mu}$ on $\bar{X}$, defined by:

$$
\mu(Y, Z)=g^{\prime \prime}\left(I_{\mu} Y, Z\right) .
$$

Let $I, J, K$ be the almost complex structures defined by $\omega^{\prime \prime}, \operatorname{Re} \eta, I m \eta$ correspondingly ( $I$ is the original complex structure on $\bar{X}$ ). Those almost complex structures satisfy

$$
I J=K, J K=I, K I=J .
$$

SLag submanifolds $L$ in $\bar{X}$ are defined by the conditions $\left.\omega^{\prime}\right|_{L}=$ $0,\left.I m \eta\right|_{L}=0$, which is of course equivalent to $\left.\omega^{\prime \prime}\right|_{L^{\prime}}=0,\left.\operatorname{Im} \eta\right|_{L^{\prime}}=0$. 
By the HyperKahler trick (see [6], p.824), this is equivalent to $L$ being a pseudoholomorphic submanifold with respect to the almost complex structure $J$.

The elements of $\Phi^{\prime}$ are $J$-holomorphic, they carry in the same homology class in $\bar{X}$ and they have a trivial self-intersection. Hence different elements of $\Phi^{\prime}$ don't intersect in $\bar{X}$. Also a neighbourhood of the boundary of $\bar{X}$ is fibered by pseudoholomorphic tori in $\Phi^{\prime}$ of the form $T_{a, b}$ (see (8)). So the elements of $\Phi^{\prime}$ cannot approach the boundary of $\bar{X}$ unless they are of the form $T_{a, b}$ (see (8)). From this it is clear that $\Phi^{\prime \prime}$ (see (9)) is the set of those elements in $\Phi$, which intersect $\bar{W}$. Also to study the subvarieties of $\bar{W}$, to which elements of $\Phi^{\prime \prime}$ degenerate it is enough to study the subvarieties of $\bar{X}$, to which elements of $\Phi^{\prime}$ degenerate.

So let $T_{i}$ be a sequence of elements in $\Phi^{\prime}$. All of those tori will carry the same homology class $h$ in $\bar{X}$. We can assume that $T_{i}$ stay away from the boundary of $\bar{X}$, for otherwise $T_{i}$ will be of the form $T_{a, b}$ (see (8)), and their limit will also be of the form $T_{a, b}$. The volume of each $T_{i}$ is $R e \eta(h)$. By Gromov's compactness theorem (see [12]), we can find a subsequence (which we still call $T_{i}$ ), which has a limit $T_{\infty}$, which is a cusp curve with at most 1 component being a torus and the rest are $J$-holomorphic spheres in $\bar{X}$. Suppose we have 1 component being a torus $T$. We will prove that $T$ is the only component, it is embedded and lives in $\Phi^{\prime}$.

We can represent $T$ as a composition $\alpha \circ \rho: T^{2} \mapsto \bar{X}$, where $\rho: T^{2} \mapsto T^{2}$ is a k-fold covering and $\alpha: T^{2} \mapsto \bar{X}$ is a simple curve (see [7], p. 18). Let $T^{\prime}=\alpha\left(T^{2}\right)$. Since $T$ doesn't intersect any of the $T_{a, b}$ (see (8)) we have $\left[T^{\prime}\right] \cdot h=0$. Also $h=k\left[T^{\prime}\right]+\Sigma\left[S_{i}\right]$ for some $J$-holomorphic spheres $S_{i}$. We have $\left[T^{\prime}\right] \cdot \Sigma\left[S_{i}\right] \geq 0$ with equality iff there are no $S_{i}$ (because the limiting curve $T_{\infty}$ is connected). Also since $\alpha$ is simple we get by Theorem 7.3 in [8] that $\left[T^{\prime}\right] \cdot\left[T^{\prime}\right] \geq 0$ with equality iff $T^{\prime}$ is embedded. From all this we deduce that there are no rational components and $T^{\prime}$ is embedded.

Since $T^{\prime}$ is $J$-holomorphic, it is a SLag torus in $\bar{X}$. By Lemma 3.1.1 the moduli-space $\Phi^{*}$ of SLag tori of $\bar{X}$ through $T^{\prime}$ is smooth and 2-dimensional. We claim that elements of $\Phi^{*}$ fill some neighbourhood of some point in $T^{\prime}$. Indeed let $\alpha_{1}, \alpha_{2}$ be 2 generators of $H^{1}\left(T^{\prime}, \mathbb{R}\right) \simeq T_{T^{\prime}} \Phi^{*}$ and $v_{1}, v_{2}$ be the corresponding normal deformation vector fields. Then $i_{v_{1}} \omega^{\prime} \wedge i_{v_{2}} \omega^{\prime}$ is nonzero in $H^{2}\left(T^{\prime}\right)$, so it doesn't vanish at some point $p \in T^{\prime}$. Hence $v_{1}$ and $v_{2}$ are linearly independent at $p$, so $\Phi^{*}$ fills a neighbourhood $U$ of $p$ in $\bar{X}$.

$T_{i}$ converge to $T^{\prime}$ in the distance topology, hence they intersect $U$ for $i$ large enough. So they intersect the elements of $\Phi^{*}$. These elements are $J$ holomorphic and carry the homology class $h / k$ and $h \cdot h=0$. So we deduce that $T_{i}$ are in $\Phi^{*}$. So $k=1$ and $T_{i}$ converge to $T^{\prime}$ in the $C^{\infty}$-topology. So 
$T^{\prime}$ is in $\Phi^{\prime}$.

Let $\partial \Phi^{\prime}$ be the set of those cusp curves in $\bar{X}$, to which elements of $\Phi^{\prime}$ degenerate. We saw that elements of $\partial \Phi^{\prime}$ are unions of $J$-holomorphic spheres. Let $\Sigma$ be a cusp curve in $\partial \Phi^{\prime}$. Then $\Sigma$ doesn't intersect any elements of $\Phi^{\prime}$. We want to prove that $\Sigma$ doesn't intersect any over cuspcurve in $\partial \Phi^{\prime}$. To prove that we have to understand the second homology $\mathrm{H}_{2}(\bar{X}, \mathbb{Z})$ in detail. Let $X$ be a neighbourhood of the torus $T=\left(\operatorname{Re} z_{1}=\right.$ $1 / 2$, Re $\left.z_{2}=1 / 2\right)$ in $T^{4}$ as in (7). In the quotient $X^{\prime}=X / \mathbb{Z}_{2}, T$ becomes a sphere $S$ and one easily sees that $S$ is a strong deformation retract of $X^{\prime}$, hence $[S]$ is a generator of $H_{2}\left(X^{\prime}, \mathbb{Z}\right)$. Let $X_{0}=X^{\prime}$ - singular points. From the Mayer-Vietoris sequence we deduce that the inclusion map $i$ : $X_{0} \mapsto X^{\prime}$ induces an isomorphism in second homology modulo torsion. Let $S^{\prime} \in H_{2}\left(X_{0}, \mathbb{Z}\right)$ s.t. $i_{*}\left(S^{\prime}\right)=[S]$. We have 2-tori $T_{a, b} \subset X_{0} \subset X^{\prime}$. One easily shows that the homology class $\left[T_{a, b}\right]$, which $T_{a, b}$ carries in $H_{2}\left(X^{\prime}, \mathbb{Z}\right)$ is $2 \cdot[S]$. From this we deduce that in $H_{2}\left(X_{0}, \mathbb{Z}\right)$ we also have $\left[T_{a, b}\right]=2 S^{\prime}$. So $[\operatorname{Re} \eta]\left(S^{\prime}\right)=1 / 2 \int_{T_{a, b}} \operatorname{Re} \eta=1 / 2$.

The resolution of singularities $\bar{X}$ is obtained from $X^{\prime}$ by replacing the singular points with the exceptional spheres $S_{i}$. One easily deduces from the Mayer-Vietoris sequence that the second homology $H_{2}(\bar{X}, \mathbb{Z})$ modulo torsion is freely generated by $S^{\prime}$ and $\left[S_{i}\right]$. Also $[R e \eta]\left(S^{\prime}\right)=1 / 2$ and $\int_{S_{i}} R e \eta=$ 0 . From this we easily get that if $\alpha \in H_{2}(\bar{X}, \mathbb{Z})$ and $[R e \eta](\alpha)>0$ then $[\operatorname{Re\eta }](\alpha) \geq 1 / 2$.

Let now $\Sigma \in \partial \Phi^{\prime}$ be as before. Then $\Sigma$ represents the homology class $h$, so $\int_{\Sigma} R e \eta=1$. Also the integral of Re $\eta$ on every component of $\Sigma$ is at least $1 / 2$, so $\Sigma$ has at most 2 components. Let $\Sigma^{\prime}$ be another cusp curve in $\partial \Phi^{\prime}$. Suppose $\Sigma^{\prime}$ intersects $\Sigma$. Since $h \cdot h=0, \Sigma$ and $\Sigma^{\prime}$ must have a common component. Suppose $\Sigma$ has a component $P$ which is not in $\Sigma^{\prime}$. Then $0=[P] \cdot h=[P] \cdot\left[\Sigma^{\prime}\right]>0$ - a contradiction. So $\Sigma$ and $\Sigma^{\prime}$ have the same components, and since their total number (counted with multiplicity) is at most 2 , then $\Sigma=\Sigma^{\prime}$.

Next we prove that the number of curves in $\partial \Phi^{\prime}$ is finite. As we have seen, there are 2 types of those curves:

1) A curve with 2 components $A_{i}$ and $B_{i}$. Then $0=\left[A_{i}\right] \cdot h=\left[A_{i}\right]$. $\left(\left[A_{i}\right]+\left[B_{i}\right]\right)$. Now $\left[A_{i}\right] \cdot\left[B_{i}\right]>0$, so $\left[A_{i}\right] \cdot\left[A_{i}\right]<0$.

If $A_{j}, B_{j}$ is another curve like that, then we have seen that $A_{i}$ doesn't intersect it, so in particular $\left[A_{i}\right] \cdot\left[A_{j}\right]=0$. So one easily sees that the numbers of such curves is at most $5=b_{2}(\bar{X})$.

2) A curve with 1 component (possibly with multiplicity 2). Let this curve be $k \cdot P_{i}$, where $P_{i}$ is a simple rational curve and $k \cdot\left[P_{i}\right]=h$. To study 
those $P_{i}$ we make the following observation : There is a $\mathbb{Z}_{2} \oplus \mathbb{Z}_{2}$ action on $T^{4}$ with generators

$$
\begin{aligned}
\gamma_{1}:\left(z_{1}, z_{2}\right) & \mapsto\left(z_{1}+i / 2, z_{2}\right), \\
\gamma_{2}:\left(z_{1}, z_{2}\right) & \mapsto\left(z_{1}, z_{2}+i / 2\right) .
\end{aligned}
$$

This action commutes with the $\alpha^{\prime}$ action and it induces an action on $\bar{X}$. Next we find those points in $\bar{X}$, which do not have a full orbit (of length 4 ) under the action. A point $\left(z_{1}, z_{2}\right)$ doesn't have a full orbit if it is preserved under one of $\gamma_{1}, \gamma_{2}, \gamma_{1} \circ \gamma_{2}$. Now the fixed points are:

$F i x\left(\gamma_{1}\right)=\left(\left(z_{1}, z_{2}\right):\left(z_{1}+i / 2, z_{2}\right)=\left(-z_{1}+1 / 2,-z_{2}+1 / 2\right)\right)$. These are 2 points in $\bar{X}$, disjoint from the exceptional spheres $S_{i}$. A similar analysis for $\gamma_{2}$ and $\gamma_{1} \circ \gamma_{2}$ produces 2 points for each.

The actions of $\gamma_{i}$ are structure preserving on $\bar{X}$. Moreover $\gamma_{i}$ preserve the SLag tori $T_{a, b}$ in $\bar{X}$ (see (8)). By Lemma 3.1.2 $\gamma_{i}$ leave the elements of $\Phi^{\prime}$ invariant. Hence they preserve the limiting curves $P_{i}$ (because the convergence is in particular in the distance topology).

For a limiting curve $P_{i}$, consider $\chi\left(P_{i}\right)=\left[P_{i}\right] \cdot\left[P_{i}\right]-c_{1}(\bar{X})\left(\left[P_{i}\right]\right)+2=2$. By Theorem 7.3 of [8] we can count $\chi\left(P_{i}\right)$ by adding the contributions of singular points of $P_{i}$ (which are multiple points or branch points), and each singular point gives a positive contribution. So $P_{i}$ has singular points and there are at most 2 of those. Let $x$ be a singular point of $P_{i}$. Then its orbit under the $\mathbb{Z}_{2} \oplus \mathbb{Z}_{2}$ action consists of singular points. So the orbit cannot have length 4 , so $x$ belongs to the set $D$ of 6 points with orbit of length 2 . So $P_{i}$ contains at least 2 points of the set $D$.

If $P_{j}$ is another curve of type 2), then $\left[P_{i}\right] \cdot\left[P_{j}\right]=0$, so they don't intersect. Also $P_{j}$ contains at least 2 points from the set $D$. So it is clear that the number of $P_{i}$ is at most 3. So the number of curves in $\partial \Phi^{\prime}$ is finite.

Let $\Phi$ be the moduli-space of SLag tori on $M$ as before, $F$ be its total space and $e v: F \mapsto M$ be the evaluation map (see the proof of Lemma 3.1.3). We claim that the differential of the evaluation map is an isomorphism everywhere. This is clearly true outside our 'bad' neighbourhoods. Inside a bad neighbourhood $\bar{W}$, it is enough to prove that for an element $L \in \Phi^{\prime}$, if $0 \neq v$ is in the tangent space $T_{L} \Phi^{\prime}$ then the normal variation vector field on $L$, which realizes the deformation given by $v$, does not vanish. But this deformation is a deformation of $J$-holomorphic tori, hence it follows from the standard argument that each zero of such a vector field gives a positive contribution to the first Chern class of the normal bundle of $L$, which is trivial. So the differential of $e v$ is an isomorphism everywhere, hence the image $e v(F)$ is open in $M$.

We have a compactified moduli-space $\bar{\Phi}$, with the boundary $\partial \Phi=\bar{\Phi}-\Phi$ parameterized by a finite union of circles $K_{i}$ (with each point in $K_{i}$ repre- 
senting a SLag submanifold in $M$ of the form $L \times S^{1}$ for $L$ being a cusp curve). It is clear that the image $\Gamma$ of the total space of $\partial \Phi$ under the evaluation map is contained in the image $i: N \mapsto M$ of a compact 4-dimensional manifold $N$ into $M$. Also the boundary of $e v(F)$ in $M$ is contained in $\Gamma$. By the standard transversality argument we deduce that $e v(F) \cup \Gamma=M$. Also we have seen that different elements in $\bar{\Phi}$ don't intersect in $M$. Thus we get a map $\alpha: M \mapsto \bar{\Phi}$, which is a SLag fibration according to the Definition 1.0.1.

\subsection{Holomorphic functions near SLag Submanifolds.}

In this section we examine holomorphic functions in a neighbourhood of a SLag submanifold. This setup appears, for instance, on a Calabi-Yau manifold $M$ in $\mathbb{C} P^{n}$ defined as a zero locus of a collection of real polynomials. In that case we have $L=M \bigcap \mathbb{R} P^{n}$ is a SLag submanifold of $M$. Let $P$ be some real polynomial of degree $k$ without real roots. Then for any polynomial $Q$ of degree $k$ the function $\frac{Q}{P}$ is a holomorphic function on $M$ in a neighbourhood of $L$. More generally let $L$ be a fixed point set of an antiholomorphic involution $\sigma$ of an almost Calabi-Yau manifold $M$ and let $h$ be a meromorphic function on $M$. Then $\overline{h \circ \sigma}$ is also a meromorphic function on $M$ and so is $g=h \cdot(\overline{h \circ \sigma})+1$. Also on $L g$ is real valued and $\geq 1$. So $f=1 / g$ is a holomorpic function in a neighbourhood of $L$ in $M$.

An immediate consequence of the fact that SLag submanifolds are 'Special', i.e., $\left.\operatorname{Im} \varphi\right|_{L}=0$ is the following:

Theorem 3.3.1. Let $L_{0}$ be SLag submanifold of an almost Calabi-Yau manifold $(M, \omega, \varphi)$ and $f$ be a holomorphic function in a neighbourhood of $L_{0}$ in $M$. Consider the function $\xi$ on the moduli-space $\Phi$ of SLag submanifolds through $L_{0}$,

$$
\xi(L)=\int_{L} f \operatorname{Re} \varphi
$$

Then $\xi$ is a constant function.

Proof. Consider the following $(n, 0)$-form $\mu=f \varphi$. Then $\mu$ is holomorphic, hence closed and obviously $\xi(L)=\int_{L} \mu$.

For $0<\theta<\pi$ we denote by $A_{\theta}$ an open cone in the complex plane $\mathbb{C}$ given by

$$
A_{\theta}=\left(z=r e^{i \rho} \mid r>0,0<\rho<\theta\right) .
$$


Corollary 3.3.1. Let $M$ be an almost Calabi-Yau n-fold and $f$ a holomorphic function on some domain $U$ in $M$. Let $L(t)$ be 1-parameter family of (compact) SLag submanifolds contained in $U$ and $p \in U$ a point s.t. $f(p)=0$. Suppose that the distance $d(p, L(t)) \rightarrow 0$ as $t \rightarrow 0$. Then $L(t)$ cannot be contained in in the domain $f^{-1}\left(A_{\theta}\right)$ for $\theta<\frac{\pi}{2 n}$.

Proof. Suppose $L(t)$ are contained in $W=f^{-1}\left(A_{\theta}\right)$ as above. We can find an $\epsilon>0$ s.t. $g=f^{n+\epsilon}$ is well defined an holomorphic on $W$ and $g(W) \subset A_{\frac{\pi}{2}}$. Then $h=\frac{\pi}{2 g}$ is holomorphic on $W, h(W) \subset A_{\frac{\pi}{2}}$ and for $z \in W$ close to $p$ we have $|h(z)| \geq$ const $\cdot d(z, p)^{-n-\epsilon}$.

Since $\int_{L(t)} h$ is constant in $t$ and Reh, Imh $>0$ on $L(t)$ then $\int_{L(t)}|h|$ is a-priori bounded by a constant $C$. Take now any $\delta>0$ and pick $t$ and $p_{t} \in L(t)$ s.t. $d\left(p, p_{t}\right) \leq \delta$. Consider $B=B\left(p_{t}, \delta\right) \cap L(t)$. By Theorem 2.0.1, $\operatorname{vol}(B) \geq \operatorname{const} \cdot \delta^{n}$ and on $B$ we have $|h| \geq \frac{\text { const }}{\delta^{n+\epsilon}}$. So $\int_{L(t)}|h| \geq \int_{B}|h| \geq$ const $\cdot \delta^{-\epsilon}$. Now $\delta$ was arbitrary - a contradiction.

Applying those ideas we can also get a restriction on SLag submanifolds in $\mathbb{C}^{n}$, which are asymptotic to a cone:

Lemma 3.3.1. Let $L \subset \mathbb{C}^{n}$ be a (smooth) SLag submanifold s.t. $L$ is asymptotic to a cone $\Lambda \subset \mathbb{C}^{n}$ and let $z_{1} \ldots z_{n}$ be coordinates on $\mathbb{C}^{n}$. Then $L$ cannot be contained in the cone

$$
B_{\theta}^{\delta}=\left(\left(z_{1}, \ldots, z_{n}\right)\left|z_{1} \in A_{\theta},\right| z_{1} \mid>\delta \cdot\|z\|\right)
$$

for $\delta>0, \theta<\pi / 2 n$. Here $A_{\theta}$ is given by (10).

Here by being asymptotic to a cone $\Lambda$ we mean the following : We have a 1-parameter family of submanifolds $L^{t}=(z \mid z / t \in L, 1 / 2 \leq\|z\| \leq 1)$ with boundary contained in the annulus $D=(z \mid 1 / 2 \leq\|z\| \leq 1)$. We define $L^{0}=\Lambda \bigcap D$ and $L^{t}$ is a smooth family of submanifolds of $D$ for $t \geq 0$.

Proof. Consider a 1-parameter family $\left(L_{t} \mid t>0\right)$ of SLag submanifolds in the unit ball in $\mathbb{C}^{n}$ with boundary in the unit sphere, $L_{t}=(z \mid z / t \in L,\|z\| \leq 1)$ (so $L^{t}=L_{t} \bigcap D$ ). We wish to prove that $\int_{L_{t}}\left|z_{1}\right|^{-n-\epsilon}$ is uniformly bounded in $t$ as $t \mapsto 0$ for some $\epsilon>0$. This will lead us to a contradiction as in the proof of Corollary 3.3 .1 because there are points in $L_{t}$ which converge to the origin in $\mathbb{C}^{n}$ for $t \rightarrow 0$.

We can choose an $\epsilon>0$ s.t. for $f=\pi / 2 \cdot z_{1}^{-n-\epsilon}$ both Ref, Imf $>0$ on $B_{\theta}^{\delta}$. So it is enough to prove that $h(t)=\int_{L_{t}} f$ is uniformly bounded in $t$ as 
$t \mapsto 0$. Consider

$$
Q_{t}=\left(z \mid z \in L_{t^{\prime}} \text { for } t^{\prime} \in[t, 1],\|z\|=1\right) .
$$

Then $F_{t}=L_{t} \bigcup Q_{t} \bigcup L_{1}$ is a closed cycle in $B_{\theta}^{\delta}$. Also clearly $F_{t}$ is homologous to 0 in $B_{\theta}^{\delta}$. The form $\varphi^{\prime}=f d z_{1} \wedge \ldots \wedge d z_{n}$ is a closed form on $B_{\theta}^{\delta}$, hence $\int_{F_{t}} \varphi^{\prime}=0$. From this we get that

$$
h(t)=h(1)+\int_{Q_{t}} \varphi^{\prime}
$$

The fact that $L$ is asymptotic to a cone implies that the volume of $Q_{t}$ is uniformly bounded in $t$. Also the conditions on $B_{\theta}^{\delta}$ imply that $\left\|\varphi^{\prime}\right\|$ is uniformly bounded on $Q_{t}$. Hence $|h(t)|$ is uniformly bounded.

\section{References.}

[1] M. Gross, Special Lagrangian Fibrations II - Geometry, Surveys in Differential Geometry: Differential geometry inspired by string theory, 341-404, International Press, 1999.

[2] J. Duistermaat, On global action-angle coordinates, Comm. Pure Appled Math., 33 (1980), 687-706.

[3] R. Harvey and H.B. Lawson, Calibrated Geometries, Acta Math., 148 (1982), 47-157.

[4] D. Joyce, Compact Riemannian 7-manifolds with holonomy $G_{2}, I$ and II, J. Differential Geom., 43(2) (1996), 291-328, 329-375.

[5] P. Lu, Special Lagrangian tori on a Borcea-Voisin threefold, math.DG/9902063.

[6] R.C. McLean, Deformations of Calibrated submanifolds, Comm. Anal. Geom., 6(4) (1998), 705-747.

[7] D. McDuff and D. Salomon, J-Holomorphic Curves and Quantum Cohomology, AMS University Lecture series, 6, Providence R.I., 1994.

[8] M. Micallef and B. White, The structure of branch points in minimal surfaces and in pseudoholomorphic curves, Ann. of Math. (2) 141(1) (1995), 35-85. 
[9] S. Salamon, Riemannian Geometry and Holonomy Groups, Pitman Research notes in math., 201, Longmann, Harlow, 1989.

[10] S. Salur, Deformations of Special Lagrangian submanifolds, math.DG/9906048.

[11] A. Strominger, S.T. Yau and E. Zaslow, Mirror Symmetry is T-Duality, Nucl. Phys., B476(1-2) (1996), 243-259.

[12] R. Ye, Gromov's compactness theorem for pseudoholomorphic curves, Trans. Amer. Math. Soc., 342(2) (1994), 671-694.

[13] S.T. Yau, On the Ricci curvature of a compact Kahler manifold and the complex Monge-Ampere equation I, Comm. Pure Appl. Math., 31(3) (1978), 339-411.

Massachusetts Institute of Technology

E-mail address: egold@math.mit.edu

ReCEIVEd November 11, 1999. 